\title{
Differential effects of antigens from $L$. braziliensis isolates from disseminated and cutaneous leishmaniasis on in vitro cytokine production
}

\author{
Paulo TG Leopoldo ${ }^{\dagger 1}$, Paulo RL Machado ${ }^{2}$, Roque P Almeida ${ }^{2}$, \\ Albert Schriefer ${ }^{2,3}$, Angela Giudice ${ }^{2}$, Amélia Ribeiro de Jesus ${ }^{2}$, John L Ho ${ }^{4}$, \\ Luiz Henrique Guimarães ${ }^{2}$, Olívia Bacellar ${ }^{\dagger 2}$ and Edgar M Carvalho* ${ }^{*+2}$
}

\begin{abstract}
Address: ${ }^{1}$ Departamento de Fisiologia, Universidade Federal de Sergipe, Aracaju, Sergipe, Brazil, ${ }^{2}$ Serviço de Imunologia, Hospital Universitário Prof. Edgard Santos, Instituto de Investigação em Imunologia (iii), Universidade Federal da Bahia, Salvador, Bahia, Brazil, ${ }^{3}$ Departamento de Biointeração, Instituto de Ciências da Saúde, Universidade Federal da Bahia, Salvador, Bahia, Brazil and ${ }^{4}$ Division of International Medicine and Infectious Diseases, Department of Medicine, Weill Medical College of Cornell University, New York, NY, USA

Email: Paulo TG Leopoldo - pauloleopoldo@bol.com.br; Paulo RL Machado - prlmachado@uol.com.br; Roque P Almeida - roque@ufba.br; Albert Schriefer - aschriefer@hupes.ufba.br; Angela Giudice - agiudice@ufba.br; Amélia Ribeiro de Jesus - amelia@ufba.br; John L Ho - jlhjlho@med.cornell.edu; Luiz Henrique Guimarães - luizhenrique@ig.com.br; Olívia Bacellar - olivinha@ufba.br; Edgar M Carvalho* - edgar@ufba.br

* Corresponding author †Equal contributors
\end{abstract}

Published: 25 April 2006

BMC Infectious Diseases 2006, 6:75 doi:10.1 I86/147/-2334-6-75

This article is available from: http://www.biomedcentral.com/I47/-2334/6/75

(C) 2006 Leopoldo et al; licensee BioMed Central Ltd.

This is an Open Access article distributed under the terms of the Creative Commons Attribution License (http://creativecommons.org/licenses/by/2.0), which permits unrestricted use, distribution, and reproduction in any medium, provided the original work is properly cited.

\begin{abstract}
Background: Disseminated leishmaniasis is an emerging infectious disease, mostly due to $L$. braziliensis, which has clinical and histopathological features distinct from cutaneous leishmaniasis.

Methods: In the current study we evaluated the in vitro production of the cytokines IFN- $\gamma$, TNF$\alpha$, IL-5 and IL-10 by peripheral blood mononuclear cells (PBMC) from 15 disseminated leishmaniasis and 24 cutaneous leishmaniasis patients upon stimulation with L. braziliensis antigens genotyped as disseminated leishmaniasis or cutaneous leishmaniasis isolates.
\end{abstract}

Results: Regardless of the source of $L$. braziliensis antigens, PBMC from cutaneous leishmaniasis patients produced significantly higher IFN- $\gamma$ than PBMC from disseminated leishmaniasis patients. Levels of TNF- $\alpha$ by PBMC from cutaneous leishmaniasis patients were significantly higher than disseminated leishmaniasis patients only when stimulated by genotyped cutaneous leishmaniasis antigens. The levels of IL- 5 and IL- 10 production by PBMC were very low and similar in PBMCs from both disseminated leishmaniasis and cutaneous leishmaniasis patients. The immune response of each patient evaluated by the two $L$. braziliensis antigens was assessed in a paired analysis in which we showed that $L$. braziliensis genotyped as disseminated leishmaniasis isolate was more potent than $L$. braziliensis genotyped as cutaneous leishmaniasis isolate in triggering IFN- $\gamma$ and TNF- $\alpha$ production in both diseases and IL-5 only in cutaneous leishmaniasis patients.

Conclusion: This study provides evidence that antigens prepared from genotypically distinct strains of $L$. braziliensis induce different degrees of immune response. It also indicates that both parasite and host play a role in the outcome of $L$. braziliensis infection. 


\section{Background}

Leishmania are obligate intracellular protozoa transmitted to mammals by infected sand flies. Human infection ranges from asymptomatic to tegumentary and visceral disease, with disfiguring and fatal outcomes in the most severe cases [1]. American tegumentary leishmaniasis (ATL) presents a spectrum of clinical manifestations including cutaneous (CL), mucosal (ML), disseminated (DL) and diffuse cutaneous leishmaniasis [2,3]. DL caused by $L$. braziliensis is an emerging infectious disease mainly observed in Northeastern Brazil. DL is characterized by numerous acneiform, papular, nodular and ulcerated skin lesions, distributed in two or more body parts [4-7]. Most importantly, compared to only 3\% among CL patients develop mucosal leishmaniasis, up to $28 \%$ of DL cases develop mucosal lesions, and the majority manifest mucosal disease concurrent with cutaneous lesions at disease presentation [7].

Previous studies with small numbers of DL patients have suggested that they often present a negative Leishmania skin test and that their lymphocytes produce lower levels of Th1 cytokines upon antigen stimulation than those of $\mathrm{CL}$ individuals [7]. In addition to the roles of host genetics and immune responses, evidence has been accumulated on the intra-species variability among Leishmania sp, and its importance in the development of different clinical forms of leishmaniasis [8-12]. Analyses employing techniques like multilocus enzyme electrophoresis (MLEE) and rRNA gene internal transcribed spacers PCR-RFLP (ITR) showed a high degree of polymorphism between isolates of $L$. braziliensis from different regions. Moreover, our recent analyses of the populations of parasites in the ATL endemic area of Corte de Pedra (CP), northeastern Brazil, revealed polymorphism among $L$. braziliensis isolates and associations between genotypes and disease outcome [8].

In this work to discern whether parasite factors influence outcome of infection towards one of these two forms of ATL, we assessed the production of IFN- $\gamma$, TNF- $\alpha$, IL- 5 and IL-10 through the stimulation in vitro of peripheral blood mononuclear cells (PBMC) from DL and CL patients with L. braziliensis antigens isolated from DL and CL patients.

\section{Methods}

Subjects

The patients enrolled in this study were recruited at the Corte de Pedra Health Post, situated in the southeast region of the State of Bahia, Brazil. The inclusion criteria were diagnosis of $C L$ and DL with the presence of active skin lesions, and age from 10 to 60 years. The exclusion criteria were pregnancy, AIDS and other immunosuppressive conditions, as well as the patient's desire of being excluded from the study. Participants of this study included 15 DL patients presenting with 10 or more mixed-type lesions (acneiform, papular, nodular and/or ulcerated) in two or more body parts (head, trunk, arms and legs), and 24 CL patients presenting with ulcerated lesions localized in a single body part. The diagnosis was confirmed by culture identification of Leishmania in lesion biopsy or in aspirates, or by histopathology typical of leishmaniasis and a positive Leishmania skin test.

This study has been approved by the Ethical Committee of the Hospital Universitário Professor Edgard Santos and informed consent was obtained from all prospectively enrolled patients.

\section{Parasites}

Two promastigotes of $L$ braziliensis, one from a patient with CL and the other from a patient with DL, each genotyped by RAPD protocols were used as antigen sources for the in vitro cytokine induction assays. The molecular genotyping of parasites was made by four RAPD protocols as previously described [8]. Cutaneous leishmaniasis L. braziliensis isolate belonged to $\mathrm{CL}$ enriched $L$. braziliensis clade $\mathrm{B}$ of $\mathrm{CP}$, while the DL isolate belonged to the $\mathrm{DL}$ enriched clade D of CP. Therefore, they are designated as L. braziliensis genotyped as CL or DL isolates. In a small number of patients experiments were repeated with two other isolates from different patients with CL and DL. The results were similar to these obtained with the original isolates.

\section{Isolation and cultivation of $L$ braziliensis}

The isolation of L. braziliensis was made in Navy McNeal Nicoli (NNN) blood agar overlaid with a modified Liver Infusion Tryptose (LIT) supplemented with $10 \%$ heat inactivated fetal bovine serum medium (FCS) (Sigma Chemical Co., St. Louis, MO) from samples obtained by needle aspiration of the skin lesions. The cultures from the biphasic medium were expanded for growth in Schneider's insect medium (Sigma), supplemented with 10\% heat inactivated FCS at $25^{\circ} \mathrm{C}$.

\section{Leishmania antigens}

Antigens used for in vitro stimulation of PBMC came from isolates of $L$. braziliensis from CL and DL patients. Promastigotes in the stationary-phase of growth were washed three times by centrifugation, adjusted to a concentration of $10^{9}$ in Phosphate Buffered Saline (PBS), and disrupted by eight repeated cycle of freezing $\left(-70^{\circ} \mathrm{C}\right)$ and thawing $\left(37^{\circ} \mathrm{C}\right)$, followed by ultrasonication. The supernatants were filtered at $0,45 \mathrm{vM}$ Millipore and stored at $-20^{\circ} \mathrm{C}$ until use. The protein content was determined by the Lowry method [13]. After performing a dose curve response to determine the optimal dose, both antigens were used in the concentration $(10 \mu \mathrm{g} / \mathrm{ml})$. 


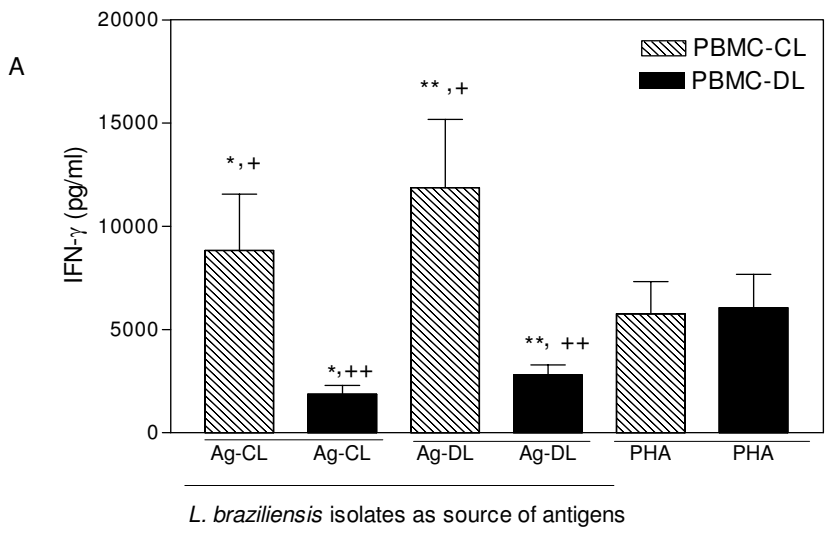

B

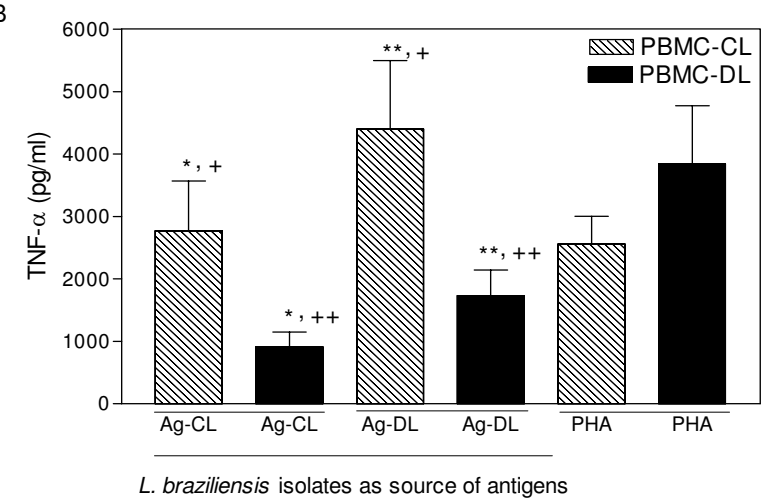

Figure I

IFN- $\gamma$ and TNF- $\alpha$ production induced by antigens from $L$. braziliensis isolates isolated CL or DL cases. PBMCs from cutaneous leishmaniasis (CL) (hatched bars, $n=$ 24) and disseminated leishmaniasis patients (black bars, $n=$ I5) were stimulated with antigens of $L$. braziliensis genotyped isolate from cutaneous leishmaniasis $(\mathrm{Ag}-\mathrm{CL})$ and disseminated leishmaniasis $(\mathrm{Ag}-\mathrm{DL})$. Data represent mean \pm standard error mean (SEM) of supernatants collected and assayed by ELISA for (A) IFN- $\gamma$, $* \mathrm{P}<0.02$ and $* * \mathrm{P}<0.0$ I, Mann-Whitney test; ${ }^{+} \mathrm{P}<0.005$ and ${ }^{++} \mathrm{P}<0.001$, Wilcoxon paired nonparametric test; and for (B) TNF- $\alpha$, $* \mathrm{P}<0.03$ and $* * P<0.8$, Mann-Whitney test; ${ }^{+},{ }^{++} \mathrm{P}<0.00 \mathrm{I}$, Wilcoxon paired nonparametric test.

\section{Separation and culture of cells}

PBMC were obtained from heparinized venous blood from patients with CL and DL by density centrifugation over a gradient of Ficoll-Hypaque (LSM; Organon Teknika corporation, Durham, NC, USA). PBMC adjusted to a concentration of $3 \times 10^{6}$ cells/ml were suspended in RPMI 1640 medium (GIBCO BRL., Grand Island, NY), 10\% heat-inactivated human $\mathrm{AB}$ serum, $2 \mathrm{mM}$ L-glutamine, $100 \mathrm{U} / \mathrm{ml}$ penicillin and $100 \mu \mathrm{g} / \mathrm{ml}$ streptomycin (GIBCO). The cells were distributed in 24-well plates and stimulated with Leishmania antigens $(10 \mu \mathrm{g} / \mathrm{ml})$ and phy- tohemaglutinin (PHA) $(100 \mu \mathrm{g} / \mathrm{ml}$, Sigma), and then incubated for $72 \mathrm{hr}$ at $37^{\circ} \mathrm{C}$ in $5 \% \mathrm{CO}_{2}$.

\section{Cytokine determination}

The supernatants were harvested and stored at $-20^{\circ} \mathrm{C}$ for cytokine determination [14]. Supernatants of cell cultures from CL and DL patients were assayed for IFN- $\gamma$, TNF- $\alpha$, IL-5 and IL-10 by sandwich ELISA technique (R \& D system, Minneapolis, $\mathrm{MN}$ ) using a mouse anti-human cytokine monoclonal antibody $(\mathrm{mAb})$ as capture antibody. Purified cytokines at varying amounts were used to derive a standard curve ( $R$ \& D system, Minneapolis, $M N$ ). The sensitivities of the cytokine dosage assays were $8 \mathrm{pg} /$ $\mathrm{ml}$ (for IFN- $\gamma$ ), $4.4 \mathrm{pg} / \mathrm{ml}$ (for TNF- $\alpha$ ), $3.9 \mathrm{pg} / \mathrm{ml}$ (for IL5) and $3.9 \mathrm{pg} / \mathrm{ml}$ (for IL-10). IL-4 was not measured because of the low sensitivity of the technique in detecting IL-4 in supernatants of PBMC from humans.

\section{Statistical analysis}

Comparison of the levels of cytokine produced by PBMC from CL and DL patients was made by the Mann-Whitney test. The paired analyses were conducted by Wilcoxon paired non-parametric test; significance was determined as $\mathrm{p}<0.05$ (two tailed).

\section{Results}

The production of IFN- $\gamma$ and TNF- $\alpha$ by peripheral blood mononuclear cells (PBMC) from CL and DL patients stimulated with antigens from L. braziliensis genotyped isolates are shown in figure 1 . In vitro IFN- $\gamma$ production by PBMC from CL patients was significantly higher than DL patients regardless of whether the source of antigen was $L$. braziliensis genotyped as a CL or DL isolate $(8810 \pm 13422$ $\mathrm{pg} / \mathrm{ml}$ versus $1849 \pm 1683 \mathrm{pg} / \mathrm{ml}$ or $11873 \pm 16184 \mathrm{pg} / \mathrm{ml}$ versus $2791 \pm 1881 \mathrm{pg} / \mathrm{ml}$, respectively; $\mathrm{P}=0.02$ or $=0.01$, respectively) (Figure $1 \mathrm{~A}$ ). TNF- $\alpha$ production by PBMC from CL patients was significantly greater than DL patients in response to $L$. braziliensis antigen from a CL isolate. Even though L. braziliensis antigen from a DL isolate triggered higher TNF- $\alpha$ production by CL patients than DL patients $(4400 \pm 5262 \mathrm{pg} / \mathrm{ml}$ versus $1729 \pm 1603$ $\mathrm{pg} / \mathrm{ml}$ ) the difference did not reach statistical significance (Figure $1 \mathrm{~B}, \mathrm{P}=0.08$ ). The observed finding of higher levels of IFN- $\gamma$ and TNF- $\alpha$ production by CL patients appeared to be specific because PBMC from CL and DL patients responded to the mitogen, PHA, by producing similar amounts of IFN- $\gamma$ and TNF- $\alpha$. We also evaluated whether the response of each patient in the specific disease group was influenced by the source of the L. braziliensis antigens. The aim was to compare IFN- $\gamma$ and TNF- $\alpha$ levels of CL patients to the two L. braziliensis antigens and the immune response of DL patients to the two L. braziliensis antigens. Using a paired analysis, L. braziliensis antigens from a DL isolate compared to a CL isolate triggered significantly greater $(\mathrm{P}<.05)$ IFN- $\gamma$ and TNF- $\alpha$ pro- 

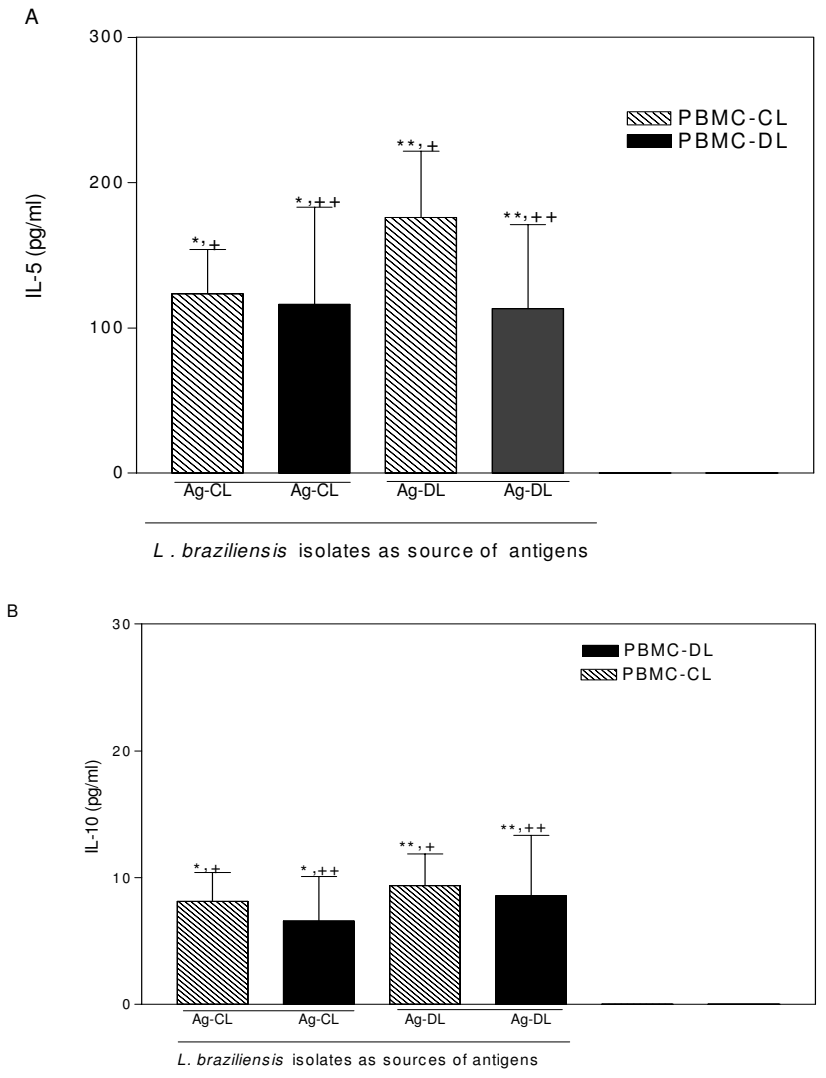

Figure 2

IL-5 and IL- 10 production induced by antigens from L. braziliensis isolates isolated CL or DL cases. Data of cutaneous leishmaniasis patients (hatched bars) and disseminated leishmaniasis patients (black bars) are illustrated as the mean \pm SEM of the same supernatants from Figure I assayed by ELISA for IL-5 $(\boldsymbol{A}) * \mathrm{P}=0.07$, **P $=0.13$, Mann-Whitney test; ${ }^{+} \mathrm{P}<0.008$ and ${ }^{++} \mathrm{P}<0.08$, Wilcoxon paired non-parametric test and IL-I0 (B) ***P $=0.4$, Mann-Whitney test; ${ }^{+} \mathrm{P}$ $=0.5$ and ${ }^{++} \mathrm{P}<0.4$, Wilcoxon paired non-parametric test.

duction by PBMC from both CL and DL patients (Figures $1 \mathrm{~A}$ e $1 \mathrm{~B})$.

We next tested IL-5 and IL-10 from the same supernatant previously assayed for IFN- $\gamma$ and TNF- $\alpha$. The levels of IL-5 and IL-10 produced by PBMC from patients with CL and DL were similar (Figure 2A). The response of each patient in the CL and DL groups to the two L. braziliensis antigens was also evaluated and showed that $L$. braziliensis antigens from a DL isolate triggered significantly higher IL-5 production than antigens from a CL isolate by PBMC from CL patients. Such a difference was not observed when tested by PBMC from DL patients (Figure 2A). Levels of IL-10 production by PBMCs from CL and DL patients were similarly low in response to antigens from $L$. braziliensis $C L$ isolate $(6 \pm 5 \mathrm{pg} / \mathrm{ml}$ and $9 \pm 14 \mathrm{pg} / \mathrm{ml}($ mean $+\mathrm{SD}))$ and L. braziliensis DL isolate $(8 \pm 12 \mathrm{pg} / \mathrm{ml}$ and $9 \pm 16 \mathrm{pg} / \mathrm{ml})$,
$\mathrm{P}=0.41$ and $\mathrm{P}=0.41$, respectively (Figure $2 \mathrm{~B}$ ). Over $50 \%$ of the CL and DL patients' PBMC cultures had undetectable levels of IL-10.

\section{Discussion}

Disseminated leishmaniasis is an emerging disease predominantly detected in the state of Bahia, Brazil $[4,5,7]$. Although $L$. amazonensis and $L$. braziliensis have been reported to be associated with DL in the past, only L. braziliensis has recently been consistently implicated with this disease [7]. Furthermore, we have recently demonstrated that certain $L$. braziliensis genotypes are more associated with DL in areas of endemicity for ATL [8]. In the current study we evaluated the production of IFN- $\gamma$, TNF- $\alpha$, IL-5 and IL-10 of PBMC from DL and CL patients, showing that DL patients produce less IFN- $\gamma$ and TNF- $\alpha$ than those with CL upon antigen stimulation, while the two groups of individuals present similar IL-5 and IL-10 secretion. In addition, we found that antigen from parasites of DL origin is a stronger inducer of the above pro-inflammatory cytokines. These data concur with results from previous work in our lab, in which we demonstrated that PBMC from DL stimulated in vitro with antigens of L. amazonensis produced lower levels of pro-inflammatory cytokines than those of CL individuals [7]. Reinforcing these findings, it has been shown that biopsies from CL patients display a higher intensity of IFN- $\gamma$ expression than those of DL individuals [15]. While lower levels of IFN- $\gamma$ and TNF$\alpha$ may contribute to the dissemination of the disease, they may also play a part in the different clinical aspects of the lesions observed in these two clinical forms of L. braziliensis. These pro-inflammatory cytokines may play key roles in the defense mechanism against Leishmania, but may also be involved in the formation of the immune mediated ulcers of CL $[16,17]$. Therefore, in contrast to the CL patients who develop large ulcers with infiltration of lymphocytes in the lesions and absent or few parasites, DL individuals present multiple lesions, frequently over 100, in different parts of their body. These lesions are mostly non-ulcerated and small, and the parasites are more easily documented [5].

Studies performed in Ethiopia on the stimulation of PBMC from CL and diffuse cutaneous leishmaniasis, a disease clinical and pathologically distinct from DL, have shown that PBMC from CL produce more IFN- $\gamma$ when stimulated with L. aethiopica antigen from CL than by antigen from diffuse cutaneous leishmaniasis. Interestingly, lymphocyte proliferation among control individuals of that endemic area was higher in response to CL antigen than diffuse cutaneous leishmaniasis antigen, supporting the idea that differences in the parasites may contribute to the clinical outcome of infection with L. aethiopica [18]. Unlike L. aethiopica, L. braziliensis does not cause diffuse cutaneous leishmaniasis and is predominantly associated 
with CL and ML diseases, characterized by a strong type 1 immune response. While indicating phenotypic variability among the strains, the observation that DL-borne $L$. braziliensis induced significantly higher levels of IFN- $\gamma$ and TNF- $\alpha$ in cultures of PBMC from CL patients was still surprising. We offer two possible explanations: (1) a bias in the innate immune response, which might be determining the outcome of DL; and (2) an over modulation of production of these cytokines by regulatory $\mathrm{T}$ lymphocytes in DL individuals with carryover effects to the in vitro evaluations. Both possibilities are currently being evaluated.

IFN- $\gamma$ and TNF- $\alpha$ are important cytokines for the outcome of infection in the experimental models of leishmaniasis. Type 1 immune responses are associated with control of infection, while type 2 leads to susceptibility for Leishmania sp [19]. In humans, low IFN- $\gamma$ production has been associated with parasite dissemination, as exemplified by VL and diffuse cutaneous leishmaniasis [20-23], in which IL-10 acts to down regulate IFN- $\gamma$ [24]. In contrast to what has been observed in VL and diffuse cutaneous leishmaniasis, we found neither a type 2 immune response nor an increase in IL-10 levels in DL patients $[23,25]$. However, it was clear the type 1 immune response is impaired in DL patients.

\section{Conclusion}

This study showed that regardless of the source of antigens, CL patients produce more IFN- $\gamma$ than DL patients. Antigens from L. braziliensis genotyped as a DL isolate was more potent than a CL isolate in promoting IFN- $\gamma$ and TNF- $\alpha$ production. This finding lends support for distinct intraspecies differences and argues for the role of intraspecies differences and host factors contributing to the clinical outcome following L. braziliensis infection.

\section{Competing interests}

The author(s) declare that they have no competing interests.

\section{Authors' contributions}

PTGL, OB and EMC participated equally in the study design, and PTGL and OB performed all the immunological dosages.

PTGL, AS, JLH and EMC drafted the manuscript.

EMC participated in the coordination of Leishmania patient evaluations.

PRLM, RPA, AS, ARJ, and LHG performed clinical evaluation of LC and LD patients.
AG isolated and cultivated Leishmania and prepared both L. braziliensis antigens.

\section{Acknowledgements}

We are grateful to Dr. Seth O'Neal for critical review of the manuscript, Elbe Myrtes Silva and Lúcia Reis for secretarial assistance. This work was supported by Fundação de Amparo à Pesquisa do Estado da Bahia (FAPESB) and Brazilian National Research Council (CNPq). Dr Edgar M. Carvalho is senior investigator of CNPq.

\section{References}

I. Pearson RD, Sousa AQ: Clinical spectrum of Leishmaniasis. Clin Infect Dis 1996, 22:I-I3.

2. Castes M, Tapia FJ: [Immunopathology of American tegumentary leishmaniasis]. Acta Cient Venez I998, 49:42-56.

3. Caceres-Dittmar G, Tapia FJ, Sanchez MA, Yamamura M, K Utyemura, Modlin RL, Bloom BR, Convit J: Determination of the cytokine profile in American cutaneous leishmaniasis using the polymerase chain reaction. Clin Exp Immunol I993, 9 I:500-5.

4. Costa JM, Marsden PD, Llanos-Cuentas EA, Netto EM, Carvalho EM, Barral A, Rosa AC, Cuba CC, Magalhaes AV, Barreto AC: Disseminated cutaneous leishmaniasis in a field clinic in Bahia, Brazil: a report of eight cases. J Trop Med Hyg 1986, 89:319-23.

5. Carvalho EM, Barral A, Costa JM, Bittencourt A, Marsden P: Clinical and immunopathological aspects of disseminated cutaneous leishmaniasis. Acta Trop 1994, 56:315-25.

6. Galvao CE, Silva AC, Saldanha AC, Silva CM, Costa Mdo R, Costa JM: [Disseminated cutaneous leishmaniasis due to Leishmania viannia braziliensis in the state of Maranhao, Brazil]. Rev Soc Bras Med Trop 1993, 26:121-3.

7. Turetz ML, Machado PR, Ko Al, Alves F, Bittencourt A, Almeida RP, Mobashery N, Johnson WD Jr, Carvalho EM: Disseminated leishmaniasis: a new and emerging form of leishmaniasis observed in northeastern Brazil. J Infect Dis 2002, I 86: 1829-34.

8. Schriefer A, Schriefer AL, Goes-Neto A, Guimaraes LH, Carvalho LP, Almeida RP, Machado PR, Lessa HA, de Jesus AR, Riley LW, Carvalho EM: Multiclonal Leishmania braziliensis population structure and its clinical implication in a region of endemicity for American tegumentary leishmaniasis. Infect Immun 2004, 72:508-14.

9. Saravia NG, Weigle K, Navas C, Segura I, Valderrama L, Valencia AZ Escorcia B, McMahon-Pratt D: Heterogeneity, geographic distribution, and pathogenicity of serodemes of Leishmania viannia in Colombia. Am J Trop Med Hyg 2002, 66:738-44.

10. Grimaldi G Jr, David JR, McMahon-Pratt D: Identification and distribution of New World Leishmania species characterized by serodeme analysis using monoclonal antibodies. Am J Trop Med Hyg 1987, 36:270-87.

II. Cupolillo E, Brahim LR, Toaldo CB, de Oliveira-Neto MP, de Brito ME, Falqueto A, de Farias Naiff M, Grimaldi G Jr: Genetic polymorphism and molecular epidemiology of Leishmania (Viannia) braziliensis from different hosts and geographic areas in Brazil. J Clin Microbiol 2003, 4I:3 I 26-32.

12. Cupolillo E, Grimaldi G Jr, Momen H: Genetic diversity among Leishmania (Viannia) parasites. Ann Trop Med Parasitol 1997, 91:6I7-26.

13. Lowry DH, G RN, Farr AL, J RR: Protein measurement with the folin phenol reagent. Journal of Biological Chemistry I95I, 193:265-275.

14. Carvalho EM, Johnson WD, Barreto E, Marsden PD, Costa JL, Reed $\mathrm{S}$, Rocha $\mathrm{H}$ : Cell mediated immunity in American cutaneous and mucosal leishmaniasis. J Immunol 1985, I 35:4|44-8.

15. Vieira MG, Oliveira F, Arruda S, Bittencourt AL, Barbosa AA Jr, Barral-Netto $M$, Barral $A$ : B-cell infiltration and frequency of cytokine producing cells differ between localized and disseminated human cutaneous leishmaniases. Mem Inst Oswaldo Cruz 2002, 97:979-83.

16. Follador I, Araujo C, Bacellar O, Araujo CB, Carvalho LP, Almeida RP, Carvalho EM: Epidemiologic and immunologic findings for the subclinical form of Leishmania braziliensis infection. Clin Infect Dis 2002, 34:E54-8.

17. Machado P, Araujo C, Da Silva AT, Almeida RP, D'Oliveira A Jr, Bittencourt A, Carvalho EM: Failure of early treatment of cutane- 
ous leishmaniasis in preventing the development of an ulcer. Clin Infect Dis 2002, 34:E69-73.

18. Akuffo H, Schurr E, Andersson G, Yamaneberhan T, Britton S: Responsiveness in diffuse versus local cutaneous leishmaniasis is due to parasite differences. Scand J Immunol 1987, 26:7|7-2|

19. Heinzel FP, Sadick MD, Mutha SS, Locksley RM: Production of interferon gamma, interleukin 2 , interleukin 4 , and interleukin 10 by CD4+ lymphocytes in vivo during healing and progressive murine leishmaniasis. Proc Natl Acad Sci U S A 199I, 88:70II-5.

20. Carvalho EM, Barral A, Pedral-Sampaio D, Barral-Netto M, Badaro R, Rocha H, Johnson WD Jr: Immunologic markers of clinical evolution in children recently infected with Leishmania donovani chagasi. J Infect Dis 1992, 165:535-40.

21. Bacellar O, Brodskyn C, Guerreiro J, Barral-Netto M, Costa CH, Coffman RL, Johnson WD, Carvalho EM: Interleukin- 12 restores interferon-gamma production and cytotoxic responses in visceral leishmaniasis. J Infect Dis 1996, I73:1515-8.

22. Carvalho EM, Bacellar OA, Reed S, Barral A, Rocha H: Visceral leishmaniasis: a disease associated with inability of lymphocytes to activate macrophages to kill leishmania. Braz J Med Biol Res 1988, 21:85-92.

23. Bomfim G, Nascimento C, Costa J, Carvalho EM, Barral-Netto M, Barral A: Variation of cytokine patterns related to therapeutic response in diffuse cutaneous leishmaniasis. Exp Parasitol 1996, 84: I88-94.

24. Bacellar O, D'Oliveira A Jr, Jeronimo S, Carvalho EM: IL-IO and IL12 are the main regulatory cytokines in visceral leishmaniasis. Cytokine 2000, I2:|228-3|.

25. Carvalho EM, Badaro R, Reed SG, Jones TC, Johnson WD Jr, Jeronimo S, Carvalho EM: Absence of gamma interferon and interleukin 2 production during active visceral leishmaniasis. Cytokine 1985, 12:|228-31.

\section{Pre-publication history}

The pre-publication history for this paper can be accessed here:

http://www.biomedcentral.com/1471-2334/6/75/prepub http.//www.biomedcentral.com/1471-2334/6/75 